\title{
Costos y competitividad en la producción de bovinos carne en corral en el sur del Estado de México
}

\author{
Costs and competitiveness in the production of beef cattle \\ in feedlot in the south of the State of Mexico
}

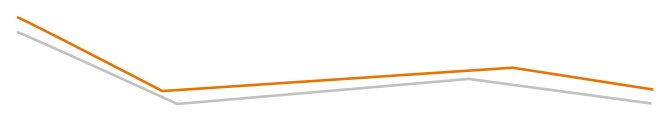

Juvencio Hernández Martínez', Alfredo Rebollar Rebollar2, Jaime Mondragón Ancelmo², Eugenio Guzmán Soria ${ }^{3}$, Samuel Rebollar Rebollar ${ }^{2 *}$

Hernández Martínez, J., Rebollar Rebollar, A., Mondragón Ancelmo, J., Guzmán Soria, E., Rebollar Rebollar, S. Costos y competitividad en la producción de bovinos carne en corral en el sur del Estado de México. Investigación y Ciencia de la Universidad Autónoma de Aguascalientes. Número 69: 13-20, septiembre-diciembre 2016.

\section{RESUMEN}

La presente investigación se realizó en el sur del Estado de México durante el periodo comprendido desde enero hasta junio de 2014. Se basó en información proveniente de 40 productores de ganado en corral, estratificados en pequeños, medianos y grandes, en donde se obtuvieron datos de ingresos y costos de producción. El objetivo de este trabajo fue analizar los costos y la competitividad de la producción bovina engordada en corral mediante la metodología de la matriz de análisis de política. Los tres tipos de productores presentaron un coeficiente de rentabilidad privada, que variaron de $10.77 \%$ a $15.40 \%$. Las relaciones de costo privado se situaron entre 0.51 y 0.52 , lo que sugiere una alta competitividad y, por tanto, permitió pagar los factores internos, incluso la tasa de retorno normal del capital, lo que dejó un margen de ganancia positivo, por lo que la actividad fue rentable y competitiva.

Palabras clave: ganancia neta, indicadores de competitividad y rentabilidad, producción bovina, rentabilidad privada.

Keywords: net profit, competitiveness and profitability indicators, cattle production, private profit.

\section{Recibido: 3 de septiembre de 2015, aceptado: 18 de julio de 2016}

Centro Universitario UAEM Texcoco, Universidad Autónoma del Estado de México.

2 Centro Universitario UAEM Temascaltepec, Universidad Autónoma del Estado de México.

3 Instituto Tecnológico de Celaya, Guanajuato.

* Autor para correspondencia: samrere@hotmail.com, srebollarr@uaemex.mx
This research was conducted in the southern State of Mexico during the period January to June 2014. It was based on information from 40 producers of cattle in feedlots, stratified in small, medium and large, where data were obtained from income and production costs. The objective of this study was to analyze production costs and competitiveness of beef production fattened in feedlots using the methodology of the policy analysis matrix. The three types of producers presented a coefficient of private profitability, which ranged from $10.77 \%$ to $15.40 \%$. Relations private cost ranged between 0.51 and 0.52 , suggesting a highly competitive, and therefore allowed to pay domestic factors, including the normal rate of return on capital, leaving a margin of positive gain, so the activity was profitable and competitive.

\section{INTRODUCCIÓN}

En México se destinan cerca de 110 millones de ha a la actividad ganadera, lo que representa alrededor de $60 \%$ del territorio nacional (FIRA, 2010), el cual incluye 1.40 millones de ranchos, corrales de engorda, empresas integrales y otras unidades económicas dedicadas principalmente a la producción de ganado bovino, en la que los sistemas de producción van desde los más altamente tecnificados e integrados hasta los tradicionales (Hernández Martínez et al., 2011). Este sector ha sido sustento para el desarrollo nacional, ya que proporciona alimentos y materias primas, divisas, 
empleos, además de que distribuye ingresos en el sector rural (Rebollar Rebollar et al., 2011).

La ganadería para carne empieza con el proceso de crianza del ganado bovino y finaliza con la engorda de las vaquillas o becerros que son destinados al mercado; posteriormente, el proceso de transformación se lleva a cabo en donde se realiza el sacrificio obteniendo la carne en canal (FIRA, 2010). Se estima que alrededor de $35 \%$ de la producción de carne de bovino en México procede de corrales de engorda (Rebollar Rebollar et al., 2011).

En 2013, los principales productores de carne bovino en canal fueron: Veracruz (13.70\%), Jalisco (11.40\%), Chiapas (6.60\%), Sinaloa (5.20\%) y Chihuahua $(4.30 \%)$, que en conjunto aportaron $45.60 \%$ del total nacional (SIAP, 2015). El Estado de México participó con un volumen de 83.90,000 t, equivalente a $2.50 \%$ de la producción nacional.

A nivel de Distrito de Desarrollo Rural (DDR), el DDR 076 de Tejupilco, Estado de México, reportó una producción de 14,830 t, concentrándose en los municipios de Tejupilco (26.50\%), Tlatlaya (19.40\%), Amatepec (17.70\%), Luvianos (15.70\%), Tejupilco (15\%), Temascaltepec (14.60\%) y San Simón de Guerrero (6.10\%), que contribuyeron con $17.70 \%$ del total estatal (SIAP, 2015). En este Distrito los sistemas de finalización de bovinos en corrales de engorda se realizan, principalmente, en explotaciones de tamaño mediano a pequeño, y la alimentación se basa en raciones de baja calidad nutricional y poco balanceadas, que se traducen en ganancias diarias de peso de aproximadamente $1.90 \mathrm{~kg} / \mathrm{d}$; además la mayoría de los productores no realizan registros sobre ingresos y costos de su actividad (Posadas Domínguez et al., 2009).

El objetivo de esta investigación fue analizar la competitividad del sistema de engorda de bovinos en corral en la región sur del Estado de México, mediante la matriz de análisis de política (MAP), con lo que se podrán establecer estrategias que faciliten a los productores aumentar su eficiencia y competitividad; la hipótesis que sustenta este trabajo es la existencia de una rentabilidad privada positiva y las relaciones de costo privado menores a la unidad, por lo que esta actividad es rentable y competitiva.

\section{MATERIALES Y METODOS}

La investigación se realizó de enero a junio de 2014, en el DDR 076 Tejupilco, Estado de México, de la Secretaría de Agricultura, Ganadería, Desarrollo Rural, Pesca y Alimentación (SAGARPA); dicho DDR incluye los municipios de Temascaltepec, San Simón de Guerrero, Tejupilco, Luvianos, Amatepec y Tlatlaya. La información provino de una encuesta dirigida (Cochran, 1984) a 40 engordadores, de un total de 60, miembros de la Unión Ganadera Regional del sur del Estado de México; quienes manifestaron disposición para proporcionar información sobre sus ingresos y costos de actividad productiva.

La estratificación de las unidades de producción seleccionadas se realizó con base en escalas (pequeños, medianos y grandes), que considera al número de animales engordados por productor. En pequeña escala están los engordadores con menos de 20 cabezas, los medianos de 20 a 49 y los grandes de 50 a 100 (Rebollar Rebollar et al., $2011)$. En el estrato de los pequeños se incluyeron 15 productores, en los medianos 18 y 7 en los grandes. Los parámetros productivos y técnicos del sistema de producción, precios, ingresos y costos, además de los coeficientes técnicos, se obtuvieron en campo y se validaron con comercializadores de alimentos balanceados y farmacéuticos (Lara Covarrubias et al., 2003; Hernández Martínez et al., 2008; Rebollar Rebollar et al., 2011).

La metodología utilizada en esta investigación fue la matriz de análisis de política (MAP), esta matriz está diseñada para identificar y cuantificar efectos de fallas de mercado y de distorsiones de política sobre precios existentes en un sistema productivo (Monke y Pearson, 1989). La MAP contiene tres filas (Tabla 1): la primera contiene los ingresos, costos y rentabilidad, medidos en precios privados; los precios privados son aquellos que, realmente, recibe o paga el productor en su actividad productiva. La segunda fila presenta los ingresos, costos y rentabilidad, medidos en precios sociales (o precios sombra). La tercera fila muestra las diferencias entre cifras de las dos primeras filas y refleja el nivel de transferencias dentro del sistema (SEPSA/FAO, 2006).

En este trabajo solamente se utilizó la información correspondiente a la primera fila, que es el presupuesto privado. En la construcción de la MAP fue necesario elaborar matrices de coeficientes técnicos, 
Tabla 1. Estructura de la matriz de análisis de política

\begin{tabular}{|c|c|c|c|c|}
\hline \multirow[b]{2}{*}{ Concepto } & \multirow[b]{2}{*}{ Ingresos totales } & \multicolumn{2}{|c|}{ Costos de producción } & \multirow[b]{2}{*}{ Ganancias } \\
\hline & & $\begin{array}{c}\text { Insumos comerciables } \\
\text { e indirectamente } \\
\text { comerciables }\end{array}$ & Factores internos & \\
\hline Precios privados & $A$ & B & C & $D$ \\
\hline Precios económicos & $E$ & $\mathrm{~F}$ & G & $\mathrm{H}$ \\
\hline Efectos de política & 1 & $\mathrm{~J}$ & K & $\mathrm{L}$ \\
\hline
\end{tabular}

Elaboración propia.

de precios de los insumos (comerciables e indirectamente comerciables), de factores internos de producción, de presupuesto y de coeficientes auxiliares.

La información se organizó y capturó en una hoja de cálculo mediante tres matrices divididas a su vez en dos apartados, insumos comerciables e indirectamente comerciables, que son bienes que se pueden importar y exportar, y los factores internos, que son bienes que no pueden comercializarse a nivel internacional. La matriz de coeficientes técnicos permitió cuantificar la tecnología de producción en cantidades de insumos, la matriz de precios privados identificó los precios que pagó y recibió, efectivamente, el productor por unidad de insumos y productos durante su proceso de producción. La matriz de presupuesto privado fue el resultado de la multiplicación de las dos anteriores, con lo que se calculó el costo de producción por componente (costo de los insumos comerciables y no comerciables y el costo de los factores internos); de esta última matriz se derivaron los análisis de ingreso y de costos, así como los principales indicadores de rentabilidad y competitividad (Tabla 2).

\section{Rentabilidad privada o ganancia (D)}

La ganancia (D) o rentabilidad privada es la retribución a la disposición del productor para administrar recursos y aceptar un riesgo (SEPSA/ FAO, 2006). Es decir, es aquella rentabilidad que, efectivamente, reciben los productores como resultado de su actividad productiva, al restar sus costos de producción, que incluyen los insumos comerciables, los indirectamente comerciables y factores internos $(B+C$ ) a sus ingresos (A) (Monke y Pearson, 1989). Una rentabilidad privada positiva significa que se es competitivo en esa actividad, lo que significa que se está obteniendo una rentabilidad superior a la normal (Hernández Martínez et al., 2008). Si las ganancias privadas son negativas, es posible que los productores abandonen esta actividad.
Tabla 2. Indicadores de rentabilidad y competitividad a precios privados

\begin{tabular}{|c|c|}
\hline Indicador & Variables \\
\hline $\begin{array}{c}\text { Rentabilidad privada o ganancia } \\
\text { Coeficiente de rentabilidad } \\
\text { privada }\end{array}$ & $\mathrm{D}=\mathrm{A}-\mathrm{B}-\mathrm{C}$ \\
\hline $\begin{array}{c}\text { Relación del costo privado } \\
\text { Valor agregado a precios } \\
\text { privados }\end{array}$ & $\mathrm{RCP}=\mathrm{C} /(\mathrm{A}-\mathrm{B})$ \\
\hline $\begin{array}{c}\text { Consumo intermedio en el ingreso } \\
\text { total }\end{array}$ & $\mathrm{VAPP}=(\mathrm{A}-\mathrm{B})$ \\
\hline Valor agregado en el ingreso total & $\mathrm{VAIT}=(\mathrm{A}-\mathrm{B}) / \mathrm{A}$ \\
\hline
\end{tabular}

Elaboración propia.

\section{Coeficiente de rentabilidad privada (CRP) y relación de costo privado (RCP)}

El CRP se utiliza para conocer la proporción del ingreso extraordinario $O$ adicional que recibe el productor por cada peso invertido, se obtiene al dividir la ganancia entre los costos de producción. La RCP señala el límite donde el sistema de producción, en términos de eficiencia, puede sostener el pago de los factores internos (incluyendo el retorno normal del capital); es decir, cuando la diferencia (A - B $C$ ) $=\mathrm{D}=0$ (Hernández Martínez et al., 2008). Si la RCP $<1$, el productor es competitivo y recibe ganancias extraordinarias; dado que después de remunerar a los factores de la producción, tanto propios como contratados, queda un residuo en el valor agregado. Si la RCP = 1, no se generan ganancias extraordinarias; entonces el productor paga, solamente, los factores de la producción. Si la RCP >1 o RCP <0 implica que el sistema productivo no permite pagar el valor de mercado de los factores internos, además de que la ganancia resulta negativa, la actividad no es redituable y no es competitivo (Sosa Montes et al., 2000). 


\section{Valor agregado a precios privados (VAPP), consumo intermedio en el ingreso total (CIIT) y valor agregado en el ingreso total (VAIT)}

El VAPP es el monto que permanece en el ingreso recibido después de haber pagado el costo de los insumos comerciables y no comerciables, sin tener en cuenta el costo de los factores internos. El CIIT son todas aquellas erogaciones destinadas a la adquisición de insumos indispensables en otros sectores de la economía. El VAIT es la remuneración de los factores internos de la producción, así como la ganancia que obtiene el productor; su valor refleja el efecto del sistema de producción hacia el interior del propio sector productivo.

\section{RESULTADOS}

En el sur del Estado de México se estima que una alta proporción del consumo regional de carne de bovino se cubre por el sistema de engorda en corral. Este sistema de producción se caracteriza porque mantiene a los animales en confinamiento por un periodo de 90 a 120 días, con una mínima inversión en infraestructura y mano de obra.

Los corrales de engorda se construyen con cercas de materiales rústicos de la misma región, tales como postes de madera, alambre de púas u otro material de desecho; los comederos, en su mayoría, son de madera de pino y de lámina de acero inoxidable; los bebederos son de tabique y cemento, a veces los construyen los propietarios de las engordas con tablas de madera y demás material rústico; mientras que los embarcaderos están hechos de postes, tablas de madera, piedra y cemento; el almacén se construye de tabique y techo de lámina de asbesto.

El ganado para engorda se adquirió en plazas públicas o tianguis regionales de ganado a un precio que fluctuó entre $27 \$ / \mathrm{kg}$ y $28 \$ / \mathrm{kg}$ en pie, con una edad de 12 y 18 meses de edad y un peso vivo (PV) de 255 a $330 \mathrm{~kg}$. Los machos adquiridos fueron cruzas de Charolais X Suizo, Simmental X Suizo y Cebú X Suizo. La engorda del ganado se basó en dietas preparadas por el productor, con ingredientes como maíz molido (30\%), sorgo (18\%), salvado (10\%), pollinaza (10\%), zacate de maíz (20\%), soya (2\%), alimento comercial (8\%) y sales minerales (2\%). El costo del $\mathrm{kg}$ de alimento preparado varió entre 4.50 y 4.80 pesos, nivel inferior al costo del alimento balanceado comercial, que se situó en 5.50.
El tiempo de engorda se efectúo entre 95 y 110 d y la ganancia promedio en kg por día fue de alrededor de $2.04 \mathrm{~kg}$. La venta del ganado ocurrió cuando el mismo alcanzó un peso entre 445 y 530 $\mathrm{kg}$ y se vendió a un precio promedio de 30 pesos en pie en corral a acopiadores regionales $y$, en menor proporción, a carniceros locales.

\section{Costos de producción privados}

La estructura de costos de producción a nivel privado para un kg de carne en pie se constituyó, en su mayor parte, por el valor de los insumos comerciables, seguido por factores internos $y$, finalmente, el de los insumos indirectamente comerciables. El costo del alimento y mano de obra abarcó los mayores porcentajes del precio total en los tres estratos de productores; $83.62 \%, 82.26 \%$ y $79.14 \%$ y la mano de obra de $7.87 \%, 9.68 \%$ y $7.49 \%$. El resto de los costos, que representan aproximadamente $10 \%$ del costo total, mismo que incluyó medicamentos, agua, fletes y maniobras, así como equipos e instalaciones, indica una limitada tecnología utilizada en esta actividad productiva (Tabla 3).

El costo promedio total de producción para los productores pequeños fue de $27.08 \$ / \mathrm{kg}$ en pie, mientras que para los medianos y grandes fue de $26.78 \$ / \mathrm{kg}$ y de $26 \$ / \mathrm{kg}$. Los costos variables estuvieron representados en alrededor de $90 \%$ con respecto al costo total en los tres estratos, donde en términos absolutos la escala de los pequeños productores registró un costo variable promedio de $24.83 \$ / \mathrm{kg}$, de los medianos $24.16 \$ / \mathrm{kg}$ y de los grandes 23.35 $\$ / \mathrm{kg}$. El costo fijo para los pequeños productores representó cerca de $8.31 \%$ del costo total, lo que en términos absolutos fue $2.25 \$ / \mathrm{kg}$, mientras que en los medianos y grandes fue de $2.62 \$ / \mathrm{kg}$ (Tabla 4).

\section{Ingreso y ganancia neta}

El precio unitario del $\mathrm{kg}$ de carne en pie fluctuó entre 28.50 y $32.50 \$ / \mathrm{kg}$, con un promedio de $\$ 30.00$, con una diferencia de $\$ 3.50$ entre el mínimo y el máximo. En los tres tipos de productores se presentaron ganancias netas positivas; pero en menor cantidad en la pequeña. En el estrato pequeño fue de $2.92 \$ / \mathrm{kg}$, en los medianos y grandes fue de 3.22 y $4 \$ / \mathrm{kg}$ de carne, cada uno (Tabla 4).

\section{Coeficiente de rentabilidad privada (CRP)}

En los tres tipos de productores se observó que por cada peso invertido el engordador obtuvo un ingreso adicional debido al manejo eficiente de los insu- 
Tabla 3. Costos de producción de bovinos en corral en el sur del Estado de México en 2014. Absoluto ( $\$ / \mathrm{kg}$ de carne) y relativo (\%)

\begin{tabular}{|c|c|c|c|c|c|c|}
\hline \multirow{2}{*}{ Concepto } & \multicolumn{2}{|c|}{ Pequeños } & \multicolumn{2}{|c|}{ Medianos } & \multicolumn{2}{|c|}{ Grandes } \\
\hline & $(\$ / k g)$ & (\%) & $(\$ / k g)$ & (\%) & $(\$ / k g)$ & (\%) \\
\hline Insumos comerciables & 24.29 & 89.68 & 23.46 & 87.61 & 22.43 & 86.28 \\
\hline Alimentación & 23.35 & 86.21 & 22.30 & 83.28 & 20.67 & 79.51 \\
\hline Vitaminas & 0.10 & 0.37 & 0.15 & 0.57 & 0.26 & 1.01 \\
\hline Desparasitantes & 0.08 & 0.28 & 0.18 & 0.68 & 0.23 & 0.88 \\
\hline $\begin{array}{l}\text { Materiales diversos (jeringas, palas, } \\
\text { carretillas, azadones) }\end{array}$ & 0.16 & 0.58 & 0.41 & 1.54 & 0.49 & 1.88 \\
\hline Combustible & 0.61 & 2.25 & 0.41 & 1.54 & 0.78 & 2.99 \\
\hline Factores internos & 2.43 & 8.96 & 2.69 & 10.05 & 3.17 & 12.21 \\
\hline Mano de obra & 1.74 & 6.42 & 1.98 & 7.39 & 2.05 & 7.89 \\
\hline Agua & 0.15 & 0.55 & 0.01 & 0.05 & 0.18 & 0.69 \\
\hline Documento de propiedad & 0.25 & 0.92 & 0.43 & 1.60 & 0.26 & 1.00 \\
\hline Uso de báscula & 0.00 & 0.00 & 0.00 & 0.00 & 0.06 & 0.24 \\
\hline Fletes y maniobras & 0.29 & 1.08 & 0.27 & 1.01 & 0.62 & 2.38 \\
\hline Insumos indirectamente comerciables & 0.37 & 1.35 & 0.63 & 2.34 & 0.39 & 1.51 \\
\hline Vehículos & 0.00 & 0.00 & 0.14 & 0.54 & 0.04 & 0.16 \\
\hline Cercas & 0.09 & 0.34 & 0.30 & 1.11 & 0.15 & 0.58 \\
\hline Comederos & 0.04 & 0.14 & 0.04 & 0.16 & 0.15 & 0.58 \\
\hline Bebederos & 0.08 & 0.29 & 0.01 & 0.06 & 0.02 & 0.06 \\
\hline Embarcaderos & 0.00 & 0.00 & 0.06 & 0.21 & 0.01 & 0.05 \\
\hline Bodegas & 0.16 & 0.59 & 0.07 & 0.28 & 0.02 & 0.09 \\
\hline Costo por engorda & 27.08 & 100.00 & 26.78 & 100.00 & 26.00 & 100.00 \\
\hline Ingreso & 30.00 & & 30.00 & & 30.00 & \\
\hline Ganancia & 2.92 & & 3.22 & & 4.00 & \\
\hline
\end{tabular}

Elaboración propia.

Tabla 4. Estructura de costos, ingresos y ganancias por tamaño de productores en el sur del Estado de México en 2014 (\$/kg de carne) y (\%)

\begin{tabular}{|l|c|c|c|c|c|c|}
\hline \multirow{2}{*}{ Concepto } & \multicolumn{2}{c|}{ Pequeños } & \multicolumn{2}{c|}{ Medianos } & \multicolumn{2}{c|}{ Grandes } \\
\cline { 2 - 7 } & $\mathbf{( \$ / \mathbf { k g } \text { de carne) }}$ & $\mathbf{( \% )}$ & $\mathbf{( \$ / \mathbf { k g } \text { de carne) }}$ & $\mathbf{( \% )}$ & $\mathbf{( \$ / k g ~ d e ~ c a r n e ) ~}$ & $\mathbf{( \% )}$ \\
\hline Costo total & 27.08 & 100.00 & 26.78 & 100.00 & 25.97 & 100.00 \\
\hline Costos variables & 24.83 & 91.69 & 24.16 & 90.22 & 23.35 & 89.90 \\
\hline Costos fijos & 2.25 & 8.31 & 2.62 & 9.78 & 2.62 & 10.10 \\
\hline Ingreso total & 30.00 & & 30.00 & & 30.00 & \\
\hline Ganancia neta & 2.92 & & 3.22 & & 4.00 & \\
\hline
\end{tabular}

mos variables, calidad del ganado y mercado de destino. Para los productores pequeños fue el más bajo con un valor de $10.77 \%$ por cada peso invertido, para los medianos $12.04 \%$ y para los grandes $15.40 \%$ (Tabla 5).

\section{Relación de costo privado (RCP)}

Los tres tipos de escalas de engordadores tuvieron una RCP mayor a cero pero menor a uno, lo cual indicó que estos productores son competitivos y reciben ganancias extraordinarias. Las RCP de los tres estratos son muy similares, ya que se ubicaron entre 0.51 y 0.52 (Tabla 5). 
Tabla 5. Resumen del presupuesto a precios privados o de mercado por tamaño de productores en el sur del Estado de México en 2014

\begin{tabular}{|l|c|c|c|}
\hline \multicolumn{1}{|c|}{ Concepto } & Pequeños & Medianos & Grandes \\
\hline Ganancia (\$/kg de carne en pie) & 2.92 & 3.22 & 4.00 \\
\hline Coeficiente de rentabilidad privada (\%/peso invertido) & 10.77 & 12.04 & 15.40 \\
\hline Relación del costo privado & 0.51 & 0.52 & 0.51 \\
\hline Valor agregado a precios privados (\$) & 5.34 & 5.91 & 7.18 \\
\hline Consumo intermedio en el ingreso total (\%) & 82 & 80 & 76 \\
\hline Valor agregado en el ingreso total (\%) & 18 & 20 & 24 \\
\hline
\end{tabular}

Elaboración propia.

\section{Valor agregado a precios privados (VAPP)}

En los tres tipos de productores existió un remanente en el ingreso total después de haber cubierto el costo de los insumos comerciables y no comerciables por la venta de un kg de carne en pie. Es decir, en el estrato de los productores pequeños, después de pagar el costo de los insumos, hubo un diferencial en el ingreso recibido por la venta de $1 \mathrm{~kg}$ de carne de $5.34 \$ / \mathrm{kg}$, necesario para el pago de mano de obra y la ganancia del productor, cantidad que fue menor con respecto a los medianos y grandes (5.91 y $7.18 \$ / \mathrm{kg})$. Esa diferencia de valores se debió a que el costo de los insumos comerciables y los indirectamente comerciables de los pequeños productores $(24.66 \$ / \mathrm{kg})$ fue mayor que el de los medianos y grandes (24.09 y $22.82 \$ / \mathrm{kg}$ ) (Tabla 5).

\section{Valor agregado en el ingreso total (VAIT)}

EI VAITP varió de 18 a $24 \%$ con respecto al ingreso total, lo que significa la mínima participación en la generación de empleo de esta actividad productiva en la región, ese porcentaje se utilizó para el pago o remuneración de factores internos de la producción (mano de obra y agua principalmente), así como la ganancia del productor, de tal manera que este valor refleja el efecto del sistema de producción hacia el interior del propio sistema (Tabla 5).

\section{Consumo intermedio en el ingreso total (CIIT)}

Este indicador se ubicó entre 76 y $82 \%$, lo que indica que la mayor parte del ingreso generado por esta actividad no se queda en la región, sino que se va hacia otro sector de la economía por concepto de adquisición de insumos comerciables, principalmente alimento. El valor más elevado en los pequeños productores indica que disponen de una mayor proporción de su ingreso para la compra de alimento en relación con los productores medianos y grandes (Tabla 5).
DISCUSIÓN

En la región sur del Estado de México la producción de ganado bovino para engorda se obtiene principalmente de la ganadería semiextensiva; sin embargo, la producción de carne de ganado bovino engordado en corral es un sistema que está empezando a cobrar importancia y abastece una buena proporción de carne en esa zona (Posadas Domínguez et al., 2009). Por su parte, Rebollar Rebollar et al. (2011) señalan que esto se debe al bajo costo de producción, ya que en este proceso productivo la infraestructura que se utiliza es de materiales e insumos de la región (Figura 1).

En este trabajo los costos de producción se situaron entre 25.97 y $27.08 \$ / \mathrm{kg}$, el más elevado fue para los productores pequeños y el más bajo para productores grandes; comportamiento similar a lo reportado por Rebollar Rebollar et al. (2011) e indicó para 2009 una oscilación entre $7.87 \$ / \mathrm{kg}$ y $20.09 \$ / \mathrm{kg}$. La disminución de los costos, a medida que aumenta el tamaño de hato, obedece a la diferencia en productividad que existe entre estratos, la cual al ser mayor con el tamaño de la explotación provoca que el costo unitario sea menor (Gamboa Mena et al., 2005; Rebollar Rebollar et al., 2011).

Los costos de producción se integraron en su mayor parte por insumos comerciables, mismos que abarcaron entre 86.28 y $89.68 \%$ de los costos totales de producción, en donde el rubro alimentación representó entre 79.51 y $86.21 \%$ de ese total; estos porcentajes difieren de los estimados por Rebollar Rebollar et al. (2011) que fluctuaron entre $85.32 \%$ y $86.12 \%$, en que la alimentación tuvo una participación de $79.14 \%$ y $83.62 \%$. La alta injerencia de la alimentación dentro de la estructura de costos indica la importancia que tiene en esta actividad productiva 
y que su análisis es fundamental en la elaboración de estrategias en el manejo de la alimentación del ganado, al optimizar el uso de estos insumos y con ello lograr ganancias aceptables que abonen a que estos sistemas de producción sean rentables y sostenibles (Posadas Domínguez et al., 2009).

En la estructura de costos de este trabajo, los variables tuvieron una participación entre 89.90 y $91.69 \%$, mientras que el porcentaje restante fueron costos fijos; esta proporción de costos variables fue menor en comparación con los estimados por Rebollar Rebollar et al. (2011), los cuales se situaron entre 93.55\% y $96.39 \%$; mientras que Gamboa et al. (2005) estimaron para Yucatán costos variables que fluctuaron de $87.3 \%$ a $94 \%$, dejando el resto para los costos fijos. Los resultados analizados indican la poca participación del costo fijo en la producción bovina bajo este sistema, lo que refleja una limitada inversión en infraestructura y en tecnología en su producción (Gamboa Mena et al., 2005; Rebollar Rebollar et al., 2011 ).

En el presente estudio las ganancias de los productores fluctuaron entre 2.92 y $4.00 \$ / \mathrm{kg}$, destacan las ganancias mayores en los productores grandes y las menores en productores pequeños; comportamiento similar, aunque con valores diferentes, fueron las ganancias que reportaron Rebollar Rebollar et al. (2011) para 2009, los cuales variaron entre 0.91 y $2.84 \$ / \mathrm{kg}$. Por su parte, Guevara et al. (2013) en una explotación intensiva de ganado bovino en Chiapas encontraron ganancias de alrededor de $3.00 \$ / \mathrm{kg}$ y señalaron que se trata de sistemas de producción ganadera sostenibles en esa zona de estudio. En el caso particular del sur del Estado de México las ganancias elevadas que tuvieron los productores grandes se debió principalmente a que en la comercialización de su ganado han mostrado una gran capacidad en la negociación del precio de venta del ganado en pie (Rebollar Rebollar et al., 2011). Además, en el proceso productivo tienen la ventaja de que cuentan con el equipo necesario para elaborar sus dietas (Posadas Domínguez et al., 2009) y, al mismo tiempo, en la compra de los insumos, la realizan adquiriendo grandes volúmenes, con lo que logran mejores precios de la materia prima.

En relación con el coeficiente de rentabilidad privada, en este trabajo se encontró que las ganancias por pesos invertidos fluctuaron entre 10.77 y $15.40 \%$ y fue más elevado en el estrato de productores grandes, tendencia similar a la que

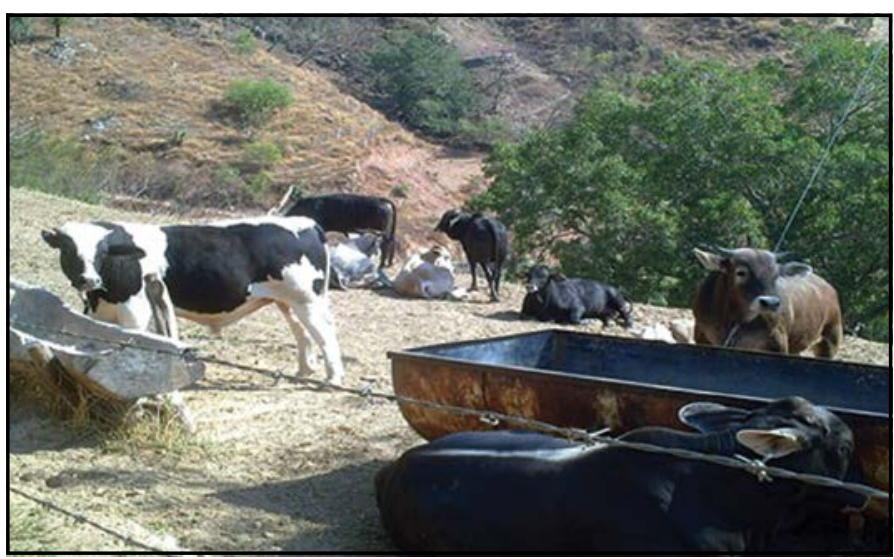

Figura 1. Engorda de bovinos en corral en el sur del Estado de México. Fotografía tomada por Samuel Rebollar Rebollar.

estimaron Rebollar Rebollar et al. (2011), entre 4.68 y 16.34\%; sin embargo, difiere con González Pérez y Gómez Monge (2014) en Michoacán, de 23 y 30 centavos por peso invertido, nivel elevado con relación al que se obtuvo en este trabajo. Ello puede deberse a la ventaja del sistema silvapastoril intensivo que radica en que el costo de producir alimentación para los bovinos es bajo por el uso de follajes naturales de pastoreo, de allí su alta rentabilidad (González Pérez y Gómez Monge, 2014).

En la presente investigación las relaciones de costo privado (RCP) de los tres tipos de productores oscilaron entre 0.51 y 0.52 , niveles menores a los que obtuvieron Rebollar Rebollar et al. (2011), que se situaron entre 0.50 y 0.79 ; esta baja en los valores de la RCP significa un aumento de la competitividad (Monke y Pearson, 1989; Hernández Martínez et al., 2008). Por su parte, González Pérez y Gómez Monge (2014) encontraron en el sistema silvopastoril intensivo una RCP que varió entre 0.40 y 0.50; este último valor es similar a las estimaciones obtenidas en esta contribución, lo que indica una alta competitividad.

Con respecto al valor agregado cuantificado, osciló entre 18 y $24 \%$, el consumo intermedio tuvo valores de 76 y $88 \%$, mientras que para estos mismos conceptos Rebollar Rebollar et al. (2011) reportaron entre 15 y $23 \%$ y entre 77 y $85 \%$; en tanto que González Pérez y Gómez Monge (2014) ubicaron el consumo intermedio en 61\%. Los bajos porcentajes de valor agregado y el alto consumo intermedio indican la poca participación en la generación de empleo sectorial, así como una gran dependencia de los otros sectores de la economía, principalmente en la 
compra de insumos comerciables e indirectamente comerciables.

\section{CONCLUSIONES}

Lasrelaciones de costo privado (RCP) enlostresestratos de productores de ganado bovino engordado en el sur del Estado de México se ubicaron en valores que indicaron una alta competitividad, lo que significa que los mismos tuvieron un mayor ingreso recibido por la venta de su producto y un menor costo de obtención en su actividad productiva. Con lo anterior se asegura que la ganancia y rentabilidad privadas de los productores fueron positivas, situación que fortaleció la eficiencia y competitividad, por lo que este sistema es una opción productiva sostenible en esta región de la entidad.

El bajo valor agregado (VAPP) generado en esta actividad, así como el alto consumo intermedio que se tiene en este sistema de producción, demandan establecer estrategias que procuren disminuir esta tendencia. El aumento del valor agregado se logrará únicamente a través del establecimiento de actividades que conduzcan a la transformación industrial del producto; en tanto que para auxiliar la disminución del alto consumo intermedio se tendrán que introducir programas regionales que promuevan la generación local de insumos, como producción de sorgo, insumo importante utilizado en la elaboración de dietas, con lo que disminuirá la transferencia de valor hacia otros sectores económicos fuera de la región.

Finalmente, la engorda de ganado bovino en corral en la región sur del Estado de México continúa siendo una actividad rentable y competitiva debido a los altos ingresos recibidos por la venta de su ganado y al abatimiento de costos en la elaboración de las dietas, así como a la mayor capacidad de negociación en la venta y a la generación de economías de escala; esto último principalmente en los productores grandes. Para fortalecer la permanencia y sustentabilidad de este sistema de producción en esta zona se debe promover el uso eficiente de materiales del productor, el cual se debe complementar con estrategias adecuadas para comercialización del ganado, entre otros aspectos.

LITERATURA CITADA

- COCHRAN, W. G. Técnicas de muestreo. D. F., México: C.E.C.S.A., 1984.

- FIRA (Fideicomisos Instituidos en Relación con la Agricultura). Panorama Agroalimentario. Carne de Bovino, 2010. Dirección General Adjunta de Inteligencia Sectorial. D. F., México: Autor, 2010.

- GAMBOA MENA, J. V. et al. Eficiencia económica de los sistemas de producción de carne bovina en el Municipio de Tizimín, Yucatán, México. Tropical and Subtropical Agroecosystems, 5, 79-84, 2005.

- GONZÁlez PÉREZ, J. M. y GÓMEZ MONGE, R. Análisis de competitividad y rentabilidad en una engorda de toretes en dos sistemas de alimentación. INCEPTUM, IX(17): 137-155, 2014.

- GuevarA, F. et al. Balance energético del sistema local de producción de bovinos de engorde en Tecpatán, Chiapas, México. Revista Cubana de Ciencia Agrícola, 47(4): 359-365, 2013.

- HERNÁNDEZ MARTínEZ, J. et al. La cadena productiva de ganado bovino en el sur del estado de México. Revista Mexicana de Agronegocios, XV(29): 672-680, 2011.

- HERNÁNDEZ MARTíNEZ, J. et al. Rentabilidad privada de las granjas porcinas en el sur del Estado de México. Universidad y Ciencia, 24(2): 117-124, 2008.

- LARA COVARRUBIAS, D. et al. Competitividad y ventajas comparativas de los sistemas de producción de leche en el estado de Jalisco, México. Agrociencia, 37, 85-94, 2003.
MONKE, E. y PEARSON, S. The Policy Analysis Matrix for Agricultural Development. Ithaca, US: Cornell University Press, 1989.

- POSADAS DOMínGUEZ, R. R. et al. Eficiencia económica en bovinos carne engordados en corral, en el sur del Estado de México. En V. B. CAVALLOTI, A. C. MARCOF y V. B. RAMÍREZ, Ganadería y seguridad alimentaria en tiempo de crisis. Vol. III (pp. 157-166). Chapingo: Universidad Autónoma Chapingo, 2009.

- RebOlLar Rebollar, A. et al. Competitividad y rentabilidad de bovinos en corral en el sur del estado de México. Tropical and Subtropical Agroecosystems, 14, 691-698, 2011.

- SEPSA (SECRETARÍA EJECUTIVA DEL SECTOR AGROPECUARIO)/ FAO (ORGANIZACIÓN DE LAS NACIONES UNIDAS PARA LA ALIMENTACIÓN Y LA AGRICULTURA). Estudio de competitividad de la porcicultura en Costa Rica con la metodología de la matriz de análisis de política (MAP). Costa Rica, 2006.

- SOSA MONTES, M. et al. Rentabilidad de doce granjas porcícolas en la región noroeste de Guanajuato en 1995. Agrociencia, 34(1): 107-113, 2000.

\section{De páginas electrónicas}

- SIAP (SERVICIO DE INFORMACIÓN AGROALIMENTARIA Y PESQUERA). Anuario estadístico de la producción agrícola de los Estados Unidos Mexicanos. 2015. Recuperado el 12 de enero de 2015, de http://www.siap.sagarpa.gob.mx/ 